\title{
Associations of measures of lung function with insulin resistance and Type 2 diabetes: findings from the British Women's Heart and Health Study
}

\author{
D. A. Lawlor · S. Ebrahim • G. Davey Smith \\ Department of Social Medicine, University of Bristol, Bristol, UK
}

\begin{abstract}
Aims/hypothesis. The aim of this study was to assess the associations of lung function with insulin resistance and Type 2 diabetes.

Methods. We did a cross-sectional study of 3911 women who were 60 to 79 years old from 23 British towns, assessing the association of measures of lung function with insulin resistance (based on fasting insulin and glucose concentrations) and Type 2 diabetes (World Health Organisation diagnostic criteria).

Results. Forced expiratory volume in 1 second (FEV1) and forced vital capacity (FVC) were inversely associated with insulin resistance and prevalence of Type 2 diabetes. In age-adjusted analyses, the homeostasis model assessment (HOMA) score (insulin resistance) decreased by $5 \%$ (95\% CI: $2-7 \%$ ) for a one standard deviation increase in log FEV1 and by $8 \%$ (95\% CI: 6-10\%) for a one standard deviation increase in log FVC. With additional adjustment for height, smoking,
\end{abstract}

BMI, waist-to-hip ratio, physical activity, white cell count, adult social class, childhood social class and respiratory medication, these associations attenuated to $3 \%(95 \%$ CI: 1 to $5 \%$ ) and 5\% (95\% CI: 3 to $8 \%$ ). The fully adjusted odds ratio for diabetes prevalence was 0.85 (95\% CI: $0.74-0.98)$ for a one standard deviation increase in log FEV1 and 0.80 (95\% CI: $0.70-0.92$ ) for a one standard deviation increase in log FVC. Forced expiratory flow in the central period of FVC was not associated with insulin resistance or diabetes.

Conclusions/interpretation. Lung function measures which predominantly reflect lung volume are inversely associated with insulin resistance and Type 2 diabetes. These associations could reflect childhood exposures which affect lung growth and also programme insulin resistance. [Diabetologia (2004) 47:195-203]

Keywords Lung function - Insulin resistance $\cdot$ Type 2 diabetes $\cdot$ Childhood exposures $\cdot$ Lung growth
Received: 14 April 2003 / Revised: 5 September 2003

Published online: 24 December 2003

C) Springer-Verlag 2003

D. A. Lawlor (

Department of Social Medicine, University of Bristol,

Canynge Hall, Whiteladies Road,

Bristol, BS8 2PR UK

E-mail: d.a.lawlor@bristol.ac.uk

Abbreviations: FEV1, Forced expiratory volume in 1 second . FVC, forced vital capacity $\cdot$ FEF25-75, forced expiratory flow in the central period of forced vital capacity .

HOMA, Homeostasis model assessment · WHO, World Health Organization
Poor adult lung function is associated with reduced survival and an increased risk for cardiovascular disease independently of smoking and other potential confounding factors $[1,2,3]$. The association could, in part, reflect an association between adverse early life exposures which affect lung growth and development and also programme adult disease $[4,5,6,7,8$, $9,10]$. Whether the association between early life exposures and later adult cardiovascular disease is driven by genetic or environmental factors, or both, insulin resistance seems to be an important intermediary in this association $[11,12,13,14]$. If the association between poor lung function and an increased risk of cardiovascular disease is partly explained by early life exposures which affect lung growth and in- 
sulin resistance, one would expect poor lung function to be associated with increased insulin resistance [15].

Though several studies have found an association between poor lung function and diabetes, fasting glucose or insulin resistance $[16,17,18,19,20,21]$, most included only a small number of participants, and several did not include non-diabetic people for appropriate comparisons [17, 19]. Furthermore, few studies have assessed a range of lung function measures. If it is correct that early life factors which affect lung growth also programme insulin resistance, one would expect a specific association between measures of lung function largely reflecting lung volume or size and insulin resistance.

Our aim was to examine the association of lung function with insulin resistance and Type 2 diabetes in a large sample of post-menopausal women. Moreover, we aimed to explain possible mechanisms for this association by examining whether a specific association with measures of lung size exists, and by controlling for potential confounding or intermediary variables.

\section{Subjects and methods}

\section{Participants}

This is a cross-sectional examination of baseline data collected for the British Women's Heart and Health Study. Full details of the selection of participants and measurements have been reported $[13,22]$. In brief, women between 60 and 79 years of age were randomly selected from general practitioner lists in 23 British towns. A total of 4286 women $(60 \%$ of the invited 7173) participated and baseline data (self-completed questionnaire, research nurse interview, physical examination and primary care medical record review) were collected between April 1999 and March 2001. Local ethics committee approvals were obtained.

\section{Measurements}

Lung function tests. Lung function tests were carried out using a spirometer (Vitalograph spirometer, Vitalograph, Ennis, Co Clare, Ireland) with an attached printout of forced vital capacity (FVC), forced expiratory volume in one second (FEV1) and forced mid-expiratory flow rate (FEF25-75). FVC is the maximum volume of air expired during forced expiration and is primarily an indicator of lung volume [23]. FEV1 is the volume of air expired in the first second of forced expiration and is influenced by lung volume and airflow obstruction [23]. FEF25-75 measures forced expiratory flow rate during the middle two quarters of forced expiration and is more sensitive than FEV1 to airflow obstruction in the smaller peripheral airways [23].

The spirometer was calibrated each day using a 1-1 syringe and automated so that results were adjusted for ambient temperature. For each assessment a research nurse demonstrated the technique to the participant, after which the participants had the opportunity to do some practice efforts. They were then required to perform a minimum of three reproducible FVC measurements (within 5\% of maximum FVC produced). The output which produced the highest sum of FVC and FEV1 was used in the analyses. Women who could not perform three reproducible measurements or who were unable to attempt the lung function assessment were excluded from the study. All assessments were carried out by one of five trained research nurses.

Insulin resistance and Type 2 diabetes. Blood samples were taken after a minimum of $6 \mathrm{~h}$ of fasting (except for diabetic patients dependent on insulin) using evacuated tubes. Plasma glucose was measured by Trinder's glucose oxidase method [24] using a Hitachi 747 and Hitachi Modular analyser (Hitachi, Kobe, Japan). Serum insulin was measured using an ELISA assay which does not cross-react with proinsulin [25]. Insulin resistance was estimated according to the homeostasis model assessment (HOMA) as the product of fasting glucose $(\mathrm{mmol} / \mathrm{l})$ and insulin $(\mu \mathrm{U} / \mathrm{ml})$ divided by the constant 22.5 [26]. As HOMA scores are a less accurate reflection of insulin resistance among older diabetic patients [27], we did not calculate HOMA scores for women with diabetes. Thus, our study assessed the associations between lung function and insulin resistance (HOMA score) among non-diabetic persons, and between lung function and diabetes prevalence in the whole cohort.

Participants completed a questionnaire in which they were asked if they had ever been diagnosed by a doctor as having diabetes, and if so, the date of diagnosis and details of treatment were requested. All participants' primary care medical records were reviewed and data extracted on diagnoses of diabetes, cardiovascular disease and cancers. A clinical diagnosis of diabetes was defined as anyone with a self-report of a doctor diagnosis or anyone identified from their medical records as having diabetes (97\% of those who fulfilled this criteria were identified both by medical records and by self-report). Type 2 diabetes was distinguished from Type 1 diabetes on the basis of age at onset and insulin treatment. All women who were diagnosed at an age younger than 30 years and who were also using insulin were defined as having Type 1 diabetes. Type 2 diabetes was defined according to WHO criteria for epidemiological studies as any woman with a clinical diagnosis of Type 2 diabetes and/or with a fasting glucose concentration of more than or equal to $7 \mathrm{mmol} / \mathrm{l}$ [28].

Other variables. Total cholesterol, HDL cholesterol and triglycerides were measured on frozen serum samples using a Hitachi 747 analyser (Hitachi, Tokyo, Japan) and standard reagents supplied by Roche diagnostics (Basel, Switzerland). A Dinamap 1846SX vital sign monitor (GE Clinical Services, Northampton, UK) was used to measure blood pressure by standard procedures. Standing and sitting height were measured, without shoes, using a Harpenden stadiometer (Critikon Service Center, Berkshire, UK) recording to the nearest millimeter. Trunk length was taken as the seated height minus the height of the stool, and leg length as the total height minus trunk length. Weight was measured in light clothing without shoes to the nearest $0.1 \mathrm{~kg}$ using Soehnle portable scales (Critikon Service Center, Berkshire, UK). Waist circumference was taken as the midpoint between the lower rib and the iliac crest. Hip circumference was taken as the largest circumference below the waist.

Details of physical activity and smoking history were obtained by self-report from either the research nurse interview or the self-completed questionnaire [22]. Adult social class was defined on the basis of the longest held occupation of the husband of married women, and the longest held occupation of the woman herself for single women. Childhood social class was defined on the basis of the longest held occupation of the participant's father. Adult and childhood social class were clas- 
sified according to the Registrar General's classification [29]. At the research nurse interview all participants brought their current medications and a detailed drugs history was undertaken. Participants were coded as taking 'respiratory medications' if they were currently using any bronchodilator or steroid medications (listed in sections $3.1-3.3$ of the British National Formulary: http://www.bnf.org/).

\section{Statistical analysis}

Analysis of variance was used to assess any variation in lung function measurements caused by patients having different nurses. Pearson's correlation coefficients were used to assess the associations between different measures of lung function among women who had never smoked and among those who were current or former smokers. To assess the direction and shape of any associations between lung function and insulin resistance, Type 2 diabetes, components of the insulin resistance syndrome and potential confounding factors, quarters of the lung function measures were used. Age-adjusted means and prevalences, and 95\% CI of each variable were estimated for these quarters using linear and logistic regression models with adjustment for an age variable which was based on the mean value of age in the whole cohort.

Multiple linear regression was used to assess the associations of each measure of lung function with insulin resistance (HOMA score) and other continuous components of the insulin resistance syndrome (HDL cholesterol, triglyceride concentrations, systolic blood pressure). In the basic models, adjustment was made for the examining nurse (included as a dummy variable), age (continuous variable) and height squared. The square of height was used because, in a detailed study of the association between stature and lung function, it was concluded that a proportional model best described the association [30]. Our fully adjusted model included all potential confounding factors (smoking, BMI, WHR, white cell count, physical activity, adult social class, childhood social class and respiratory medication) in addition to nurse, age and height squared. A similar series of multiple logistic regression models were undertaken to assess the association between measures of lung function and Type 2 diabetes.

Of the 3911 women with adequate measures of lung function, 363 could not be assigned an adult social class and 469 could not be assigned a childhood social class because they did not provide data on occupation. Although the participants were not specifically asked about unemployment, these women are likely to have been married to unemployed men (for those with missing adult data) or to have had fathers who were unemployed (for those with missing childhood data). This is consistent with findings that women without these data on social class are more likely to smoke, more likely to be obese, are shorter and more likely to have prevalent coronary heart disease than cohort members who provided these data [14]. In the main analysis, women with missing social class data were allocated to social class $\mathrm{V}$, the most deprived group. A sensitivity analysis was conducted, in which these women were excluded from the analysis. The results did not differ substantively from the main analyses and have not been presented in this paper. Because of the strong association between smoking and lung function, simple adjustment for smoking in regression models could be insufficient to account for its potential confounding effects. As a result the associations of lung function with insulin resistance and Type 2 diabetes were assessed in a subgroup of women who were lifelong non-smokers as well as being either single or partnered with life-long non-smokers.
Leg length is a specific biomarker of early life environmental exposures which affect growth, and leg length is specifically associated with insulin resistance $[13,31]$. To investigate the combined effects of lung function and leg length on insulin resistance we included measures of both in the regression models. Likelihood ratio tests were used to assess the statistical significance of the interaction terms in these models. The authors do not consider an arbitrary $p$ value of $p=0.05$ to be useful. Actual $p$ values are presented in the Results.

Lung function measurements, HOMA scores and triglyceride concentrations were positively skewed; geometric means are shown and the natural log of the values was used in the regression models. In analyses in which HOMA scores and triglyceride concentrations were the outcome, the resultant regression coefficients were back-transformed to give a ratio of geometric means. From this we calculated a proportional per unit change in lung function measure. In all analyses robust confidence intervals were estimated which take into account the clustering between participants from the same towns.

\section{Study ethics}

United Kingdom local ethics committee approvals were obtained for the British Women's Heart and Health Study. All participants included in this study gave written informed consent for their primary care medical records to be reviewed.

\section{Results}

Cohort characteristics. Compared to responders $(n=4286)$, non-responders $(n=2887)$ were older (mean age 70.2 years vs 68.9 years, $p<0.001)$ and had a higher prevalence of diabetes recorded in their primary care medical records $(4.8 \%$ vs $7.7 \%, p<0.001)$. The prevalence of coronary heart disease and cancer was similar between responders and non-responders ( $p>0.3$ for all). Of the 4286 participants, 3911 (91\%) completed adequate lung function measures. Those who were unable to complete adequate measures were older (mean age \pm SD: $69.8 \pm 5.7$ years vs $68.8 \pm 5.5$ years, $p=0.001)$ and more likely to have ever been smokers $(61.7 \%, 95 \%$ CI: $56.6-66.6 \%$ vs $53.5 \%, 95 \%$ CI: $52.0-55.1 \%, p=0.003)$. The geometric mean of HOMA scores $(1.67,95 \%$ CI: $1.45-1.96$ vs $1.66,95 \%$ CI: $1.63-1.70, p=0.85)$ and the prevalence of diabetes (9.8\%, 95\% CI: $8.9-10.8 \%$ vs $11.8 \%, 95 \%$ CI: $5.8-21.9 \%, p=0.45$ ) did not differ between those with and without adequate lung function tests.

All further analyses were conducted only on the 3911 women with lung function measures. There was evidence of variation in lung function assessments carried out by the different nurses (ANOVA for difference in means, $p=0.05$ for FEV1, $p=0.08$ for FVC and $p=0.09$ for FEF25-75). All lung function measures have therefore been adjusted for examining nurse, by including examining nurse as a dummy variable in all analyses. The geometric mean of HOMA scores among the 3911 women included in this study was 1.67 (95\% CI: 1.64-1.71) and the prevalence of WHO-defined Type 2 diabetes was $9.8 \%$ (95\% CI: 8.9-10.8\%). 
Table 1. Pearson's correlation coefficients (95\% CI) between measures of lung function among life-long non-smokers and current or former smokers

\begin{tabular}{lllll}
\hline & & Log FEV1 & Log FVC & Log FEF25-75 \\
\hline Log FEV1 & & 1 & NA & NA \\
Log FVC & Life-long non-smokers & $0.86(0.85-0.87)$ & 1 & NA \\
& Current or former smokers & $0.86(0.85-0.87)$ & & 1 \\
Log FEF25-75 & Life-long non-smokers & $0.68(0.66-0.70)$ & $0.30(0.30-0.38)$ & \\
& Current or former smokers & $0.77(0.77-0.81)$ & $0.45(0.41-0.49)$ \\
\hline
\end{tabular}

FEV1, forced expiratory volume in one second; FVC, forced vital capacity; FEF25-75, forced expiratory flow rate in mid-phase of expiration; NA, not applicable

Table 2. Age-adjusted and multivariable associations of measures of lung function with insulin resistance, components of the insulin resistance syndrome and diabetes among all participants irrespective of smoking status

Number Change $^{\mathrm{b}}$ adjusted for nurse, age and height squared $(95 \% \mathrm{CI})$
Fullyc adjusted change ${ }^{\mathrm{b}}$ $(95 \% \mathrm{CI})$

Exposure $=1$ standard deviation log FEV1

HOMA score among non-diabetics only

Diabetes prevalence

HDLc

Triglycerides

Systolic blood pressure

Exposure $=1$ standard deviation log FVC

HOMA score among non-diabetics only

Diabetes prevalence

HDLc

Triglycerides

Systolic blood pressure

Exposure $=1$ standard deviation log FEF25-75

HOMA score among non-diabetics only

Diabetes prevalence

HDLc

Triglycerides

Systolic blood pressure

a Number with complete data on all variables included in fully adjusted model

${ }^{\mathrm{b}}$ Change $=$ regression coefficients [absolute difference for HDLc and systolic blood pressure and proportionate (\%) change for HOMA score and triglycerides] for continuous variables and odds ratio for diabetes prevalence for a one standard deviation increase in $\log \mathrm{FEV} 1, \log \mathrm{FVC}$ and $\log \mathrm{FEF} 25-75$

One standard deviation of $\log \mathrm{FEV} 1=0.29$, one standard deviation of $\log \mathrm{FVC}=0.25$, one standard deviation of $\log$ FEF25-75=0.55

Table 1 shows the correlation matrix for the associations of different measures of lung function among women who had never smoked and among those who were current or former smokers. Log FEV1 and $\log$ FVC were strongly correlated, and the magnitude of this association was similar in smokers and nonsmokers. Log FEV1 was also correlated with log FEF25-75; the magnitude of this association was greater among smokers than among non-smokers (difference, $p<0.001)$. Log FEF25-75 was weakly corre-

$$
\begin{aligned}
& -5 \%(-7 \text { to }-2 \%) \\
& 0.81(0.73 \text { to } 0.91) \\
& 0.01(-0.01 \text { to } 0.03) \\
& -2 \%(-3 \text { to }-1 \%) \\
& -1.01(-1.91 \text { to }-0.11)
\end{aligned}
$$

$$
\begin{aligned}
& -3 \%(-5 \text { to }-1 \%) \\
& 0.85(0.74 \text { to } 0.98) \\
& -0.01(-0.03 \text { to } 0.01) \\
& 0 \%(-1 \text { to } 2 \%) \\
& -1.06(-2.04 \text { to }-0.08)
\end{aligned}
$$

$-8 \%(-11$ to $-6 \%)$

$0.72(0.63$ to 0.81$)$

$0.03(0.02$ to 0.05$)$

$-5 \%(-7$ to $-3 \%)$

$-1.05(-1.96$ to -0.15$)$

$$
\begin{aligned}
& -5 \%(-8 \text { to }-3 \%) \\
& 0.80(0.70 \text { to } 0.92) \\
& 0.02(0.01 \text { to } 0.04) \\
& -3 \%(-4 \text { to }-1 \%) \\
& -0.89(-1.85 \text { to } 0.02)
\end{aligned}
$$

$$
\begin{aligned}
& -1 \%(-3 \text { to } 2 \%) \\
& 0.93(0.83 \text { to } 1.04) \\
& 0.01(-0.01 \text { to } 0.03) \\
& 1 \%(-1 \text { to } 3 \%) \\
& -0.64(-1.47 \text { to } 0.19)
\end{aligned}
$$

$$
\begin{aligned}
& 0 \%(-2 \text { to } 2 \%) \\
& 0.93(0.83 \text { to } 1.05) \\
& 0.01(-0.01 \text { to } 0.02) \\
& 2 \%(-1 \text { to } 3 \%) \\
& -0.91(-1.80 \text { to }-0.02)
\end{aligned}
$$

${ }^{\mathrm{c}}$ Fully adjusted $=$ adjusted for examining nurse, age, height squared, smoking, physical activity, BMI, WHR, white cell count, respiratory medication, adult social class, childhood social class

FEV1, forced expiratory volume in one second; FVC, forced vital capacity; FEF25-75, forced expiratory flow rate in midphase of expiration; HDLc, HDL cholesterol, HOMA, homeostasis model assessment

lated with $\log \mathrm{FVC}$, and this correlation was also greater in smokers than in non-smokers $(p<0.001)$.

Associations of lung function with insulin resistance and diabetes. There was a linear inverse association of FEV1 with HOMA score, Type 2 diabetes prevalence, triglyceride concentrations and systolic blood pressure. Mean HDL cholesterol was highest in women in the highest quarter of FEV1 measures but was not linearly associated with FEV1 across the distribution. 
Table 3. Age-adjusted and multivariable associations of measures of lung function with insulin resistance, components of the insulin resistance syndrome and diabetes among women who were life-long non-smokers and were either single or had partners who were life-long non-smokers
Number ${ }^{\mathrm{a}} \quad$ Change $^{\mathrm{b}}$ adjusted for nurse, age and height squared $(95 \% \mathrm{CI})$
Fullyc adjusted changeb $(95 \% \mathrm{CI})$

Exposure $=1$ standard deviation log FEV1

HOMA score among non-diabetics only

Diabetes prevalence

HDLc

Triglycerides

Systolic blood pressure

Exposure $=1$ standard deviation log FVC

HOMA score among non-diabetics only

Diabetes prevalence

HDLc

Triglycerides

Systolic blood pressure

Exposure $=1$ standard deviation log FEF25-75

HOMA score among non-diabetics only

Diabetes prevalence

HDLc

Triglycerides

Systolic blood pressure

$$
\begin{aligned}
& -6 \%(-11 \text { to }-2 \%) \\
& 0.78(0.64 \text { to } 0.95) \\
& 0.03(0.00 \text { to } 0.05) \\
& -2 \%(-5 \text { to } 0 \%) \\
& -0.64(-2.12 \text { to } 0.83)
\end{aligned}
$$

$$
\begin{aligned}
& -9 \%(-13 \text { to }-5 \%) \\
& 0.69(0.57 \text { to } 0.82) \\
& 0.04(0.01 \text { to } 0.06) \\
& -5 \%(-8 \text { to }-3 \%) \\
& -0.61(-1.96 \text { to } 0.74)
\end{aligned}
$$

$\begin{array}{ll}1486 & -9 \%(-13 \text { to }-5 \%) \\ 3632 & 0.69(0.57 \text { to } 0.82) \\ 1677 & 0.04(0.01 \text { to } 0.06) \\ 1677 & -5 \%(-8 \text { to }-3 \%) \\ 1685 & -0.61(-1.96 \text { to } 0.74)\end{array}$

$-5 \%(-8$ to $-2 \%)$

$0.76(0.61$ to 0.93$)$

$0.02(0.00$ to 0.04$)$

$-3 \%(-5$ to $-1 \%)$

$-0.16(-1.57$ to 1.24$)$

a Number with complete data on all variables included in fully adjusted model

${ }^{\mathrm{b}}$ Change $=$ regression coefficients [absolute difference for HDLc and systolic blood pressure and proportionate (\%) change for HOMA score and triglycerides] for continuous variables and odds ratio for diabetes prevalence for a one standard deviation increase in log FEV1, log FVC and log FEF25-75 One standard deviation of $\log \mathrm{FEV} 1=0.29$, one standard deviation of $\log \mathrm{FVC}=0.25$, one standard deviation of $\log$ FEF25-75=0.55

Similarly, FVC was linearly and inversely associated with HOMA scores, diabetes prevalence, triglycerides and systolic blood pressure. The magnitude of the association of FVC with HOMA scores and diabetes was greater than those of FEV1 with HOMA scores and diabetes (difference for association with HOMA, $p=0.07$; difference for association with diabetes $p=0.02$ ). There was only a weak inverse association of FEF25-75 with HOMA scores and diabetes.

Table 2 shows the multivariable associations of each measure of lung function with insulin resistance (HOMA scores), Type 2 diabetes prevalence and components of the insulin resistance syndrome. In this table the simpler model with adjustment for age, examining nurse and height is compared with the fully adjusted model. Both models included only those participants who had complete data on all variables included in the fully adjusted model. There were small numbers of women with missing data for different variables ( $\leq 2 \%$ for any one variable). In total just over 3600 of the 3911 women with lung function data had complete data on all variables included in the fully adjusted model. Those with complete data did not differ

$$
\begin{aligned}
& 0 \%(-5 \text { to } 3 \%) \\
& 0.95(0.79 \text { to } 1.14) \\
& 0.02(-0.01 \text { to } 0.04) \\
& 1 \%(-1 \text { to } 4 \%) \\
& -0.29(-1.62 \text { to } 1.04)
\end{aligned}
$$

${ }^{\mathrm{c}}$ Fully adjusted $=$ adjusted for examining nurse, age, height squared, physical activity, BMI, WHR, white cell count, respiratory medication, adult social class, childhood social class FEV1, forced expiratory volume in one second; FVC, forced vital capacity; FEF25-75, forced expiratory flow rate in midphase of expiration; HDLc, HDL cholesterol, HOMA, homeostasis model assessment from those without complete data with respect to age or any measures of lung function ( $p>0.5$ for all). With full adjustment for all potential confounding factors, the decrease in HOMA score for a one standard deviation increase in log FEV1, adjusted for age, height and examining nurse, attenuated from 5\% (95\% CI: 2-7\%) to $3 \%$ (95\% CI: $1-5 \%)$ and the odds ratio of diabetes prevalence decreased from 0.79 (95\% CI: 0.71-0.88) to 0.85 (95\% CI: 0.74-0.98). Both, however, remained inversely associated. Similarly, for a one standard deviation increase in $\log \mathrm{FVC}$ with full adjustment for all potential confounding factors, HOMA score decreased by $5 \%$ (95\% CI: $3-8 \%$ ) and the odds ratio for diabetes prevalence was 0.80 (95\% CI: 0.70 to 0.92 ). In the fully adjusted models, FEV1 remained inversely associated with systolic blood pressure but not with other components of the insulin resistance syndrome. FVC was inversely associated with triglyceride concentrations and positively associated with HDL cholesterol concentrations in the fully adjusted models. It also showed a moderate inverse association with systolic blood pressure. The variable FEF25-75 also showed a moderate inverse association with systolic 
blood pressure, but was not associated with any other components of the insulin resistance syndrome.

Associations of lung function with insulin resistance and diabetes among life-long non-smokers. Among the 3911 women, 1817 (46\%) were life-long nonsmokers and were either single or partnered to individuals who were also life-long non-smokers. All measures of lung function were greater among these women. The geometric means of FEV1, FVC and FEF25-75 among women who were unexposed to smoking were 1.99 (95\% CI: 1.96-2.01), 2.78 (95\% CI: 2.75-2.81) and 1.39 (95\% CI: 1.36-1.43) respectively. Among women who were current or former smokers, the values were 1.83 (95\% CI: $1.80-1.85$ ), 2.66 (95\% CI: 2.63-2.69) and 1.16 (95\% CI: $1.13-1.18$ ) respectively (all differences, $p<0.001$ ). Table 3 shows the associations of measures of lung function with insulin resistance, diabetes and components of the insulin resistance syndrome among this subgroup of women with little or no life-long exposure to cigarette smoke. For most outcomes these results were not substantively different to those presented in Table 2 for the whole cohort. In particular, the inverse associations of FEV1 and FVC with insulin resistance and diabetes prevalence were the same in women who were not exposed to cigarette smoke as in the whole cohort. The inverse associations between each measure of lung function and systolic blood pressure seen in the entire cohort were all considerably weaker among the subgroup of women who were not exposed to cigarette smoke, suggesting that these associations were confounded by smoking.

The effect of birthweight on the associations of lung function with insulin resistance and diabetes. Of the 3911 women with lung function measurements, 1279 (33\%) provided self-reported details of their birthweight. Women who provided birthweight data were younger than those who did not provide these data (mean age $\pm \mathrm{SD}, 67.8 \pm 5.4$ vs $69.3 \pm 5.5, p<0.001$ ) but there was no substantive difference in age-adjusted means of HOMA score or prevalence of diabetes between these two groups ( $p>0.5$ for both). Age-adjusted lung function measurements were better among women providing these data [age-adjusted mean FEV1 among those with these data 2.00 (95\% CI: 1.98-2.04) vs 1.96 (95\% CI: $1.94-1.97)$ than among those without these data, $p=0.001]$. Among this subgroup of women with self-reported birthweight, the age and confounder-adjusted (as in final column of Table 2) change in HOMA score for a one standard deviation increase in log FEV1 was $-2 \%$ ( -7 to $1 \%$ ) and that for a one standard deviation increase in log FVC was $-7 \%$ ( -11 to $-3 \%$ ). With additional adjustment for self-reported birthweight, these values were essentially unaltered: $-3 \%$ ( -7 to $1 \%)$ for FEV1 and $-8 \%(-12$ to $-3 \%)$ for FVC. Similarly, among this subgroup of women, the age and confounder-adjusted odds ratio for Type 2 diabetes for a one standard deviation increase in FEV1 was 0.78 (95\% CI: 0.63 to 0.96 ) and that for a one standard deviation increase in FVC was 0.77 (95\% CI: 0.61 to 0.96 ). With additional adjustment for birthweight, these associations were essentially unaltered: 0.78 (95\% CI: 0.63-0.96) for FEV1 and 0.77 (95\% CI: 0.61-0.96) for FVC.

Combined effects of lung function and components of adult height. Both leg length and trunk length were positively associated with lung function. Lung function (FVC and FEV1) and leg length were both independently associated with insulin resistance and diabetes. When both were included in the same regression model (together with age and examining nurse), HOMA scores decreased by 7\% (95\% CI: 4-9\%) for each increase in standard deviation of $\log \mathrm{FVC}$ and by $3 \%$ (95\% CI: $1-6 \%$ ) for each increase of one standard deviation of leg length. For the combination of FEV1 and leg length, HOMA scores decreased by $4 \%(95 \%$ CI: $1-6 \%$ ) for each standard deviation increase in log FEV1, and by $4 \%$ (95\% CI: 2-7\%) for each standard deviation increase in leg length. For FVC and FEV1, better lung function and greater leg length combined additively to decrease insulin resistance (interaction in model with FVC and leg length, $p=0.68$; interaction in model with FEV1 and leg length, $p=0.32$ ). Similarly, lung function and leg length were independently associated with decreased odds of diabetes, and combined multiplicatively with no strong statistical evidence of an interaction. With adjustment for trunk length, the inverse associations of lung function with insulin resistance and Type 2 diabetes remained, but they were slightly attenuated. Trunk length was not independently associated with insulin resistance and diabetes when measures of lung function were included in the regression models.

\section{Discussion}

The main findings of our study were that FEV1 and FVC are inversely associated with insulin resistance and with Type 2 diabetes. These associations were still evident after adjustment for important adult confounding factors, and were also similar in a group of women with little or no lifetime exposure to cigarette smoke. FEF25-75 was not associated with insulin resistance or Type 2 diabetes.

Our response rate $(60 \%)$ was moderate but consistent with other contemporary large epidemiological surveys [32]. Respondents were slightly younger than non-respondents and less likely to have had a primary care medical record of diabetes. If our cohort is healthier than the general population of older women, it is possible that our estimates of the associations pre- 
sented are diluted. They would only be exaggerated if the associations were in the opposite direction or markedly weaker among non-responders, which seems unlikely. Our study is cross sectional and a potential limitation is survivor bias. This would only lead to results that exaggerate the true effect, if poor lung function were associated with increased insulin resistance and diabetes and also increased survival, which seems unlikely. Reverse causality could also be a problem. It is conceivable that people who become ill with diabetes reduce their physical activity and become more obese with consequent reduction in lung function. However, the association with diabetes was independent of physical activity and obesity. The inverse association with insulin resistance in non-diabetic women (who are likely to be largely asymptomatic) also suggests that this is an unlikely explanation of the association. In addition, our findings are consistent with smaller, but prospective, studies $[15,18,20,15$, 21].

For birthweight we used self-reported data, which could be inaccurate. However, self-reported birthweight is strongly correlated with hospital records among middle-aged and older women [33]. Women in our study were born between 1919 and 1940 and the mean self-reported birthweight for these women was $3.28 \mathrm{~kg}(\mathrm{SD} \pm 0.80)$ which is consistent with hospital records of women born between 1923 and 1930 in Hertfordshire, England (3.42 kg) [34], and also with women in the 1946 British cohort $(3.32 \mathrm{~kg}$, $\mathrm{SD} \pm 0.49$ — data provided by Prof. D. Kuh). Any misclassification bias is likely to have been non-differential and will therefore have diluted any effect of birthweight on the associations presented. Only one-third of the participants provided birthweight data and these tended to be younger than those without these data. However, the age-adjusted distributions of insulin resistance and diabetes did not differ between those with and without birthweight data.

We have assessed the associations of three different measures of lung function with insulin resistance and Type 2 diabetes. FVC largely reflects lung volume, whereas FEV1 is influenced by airway flow obstruction and lung volume. FEF25-75 is a more sensitive indicator of peripheral airway obstruction [23]. In the general population, most people with marked impairment of FEV1 have airway obstruction [35] and this has, to some extent, resulted in a common perception that low FEV1 is predominantly an indicator of airflow obstruction. In reality this is probably only so for the extreme low end of the FEV1 distribution, with FEV1 in general being a reflection of lung volume $[23,36]$. The finding in our study that the correlations between FEV1 and FEF25-75 and between FVC and FEF25-75 are stronger among women who had smoked at some time than among those who had never smoked supports this assertion. This is because smokers are highly likely to have seriously diseased lungs, with restriction of peripheral airway flow and lung volume, and all measures of lung function reflecting this generally reduced lung capacity. Among nonsmokers, lung volume and peripheral airway obstruction are more discrete entities. Thus, our results suggest that the factors associated specifically with lung volume are inversely associated with insulin resistance and diabetes.

The persistent associations of FVC and FEV1 with insulin resistance and Type 2 diabetes among a group of women with little or no exposure to smoking in their lives suggests that the association is not a reflection of the detrimental effect of smoking on diabetes [37]. We have also shown that the associations do not appear to be due to known adult risk factors linking poor lung function to insulin resistance and diabetes, nor do they appear to be due to the known association of low birthweight with lung function and insulin resistance. We propose that the association is, at least in part, explained by early life factors which affect lung growth and programme insulin resistance. This suggestion is supported by the specific association of measures of lung volume with insulin resistance and diabetes, and by the exclusion of other plausible explanations. It is also supported by recent findings of a prospective study which found that when adults with Type 2 diabetes were compared with non-diabetic adults they had consistently poorer lung function, but that the rate of decline in lung function with increasing age was similar in both groups $[38,39]$.

We also found an association between adult leg length and measures of lung function. Whilst a direct association between trunk length and lung volume is to be expected, a direct association between leg length and measures of lung volume is harder to explain. As leg length and trunk length are only weakly correlated with each other $[13,31]$ (Pearson's correlation coefficient $=0.26$ in this study), the association between leg length and lung function is unlikely to simply reflect the association between trunk length and lung function measures. It is possible that the association between leg length and lung function is explained by early life factors affecting lung and leg growth. However, we found that leg length and lung function had independent effects on insulin resistance and diabetes, which could be explained by different early life factors, all of which affect insulin resistance but which also have differential effects on skeletal and lung growth.

Our results suggest that early life exposures which influence lung growth also programme insulin resistance. If our suggestions are correct, they emphasise the importance of early life exposures in the development of insulin resistance and the need for programmes aimed at improving child health for the prevention of insulin resistance and its associated complications. 
Acknowledgements. The British Women's Heart and Health Study is funded by the Department of Health, UK Government. D.A. Lawlor was funded by a Medical Research Council and Department of Health research training fellowship when this work was conducted and is now funded by a Department of Health career scientist award. We thank all of the general practitioners and their staff who supported data collection, and the women who have participated in the study. Dr. M. Upton, Senior Lecturer of the Department of Social Medicine, University of Bristol, UK, commented on an earlier draft of the paper. The views expressed in this publication are those of the authors and not necessarily those of the Department of Health or Medical Research Council. We are not aware of any conflicts of interests associated with this study.

\section{References}

1. Strachan DP (1992) Ventilatory function, height, and mortality among lifelong non-smokers. J Epidemiol Community Health 46:66-70

2. Hole DJ, Watt GC, Davey Smith G, Hart CL, Gillis CR, Hawthorne VM (1996) Impaired lung function and mortality risk in men and women: findings from the Renfrew and Paisley prospective population study. BMJ 313:711-715

3. Schunemann HJ, Dorn J, Grant BJ, Winkelstein W Jr, Trevisan M (2000) Pulmonary function is a long-term predictor of mortality in the general population: 29-year follow-up of the Buffalo Health Study. Chest 118:656-664

4. Gunnell D, Whitley E, Upton MN, McConnachie A, Davey Smith G, Watt GC (2003) Associations of height, leg length, and lung function with cardiovascular risk factors in the Midspan Family Study. J Epidemiol Community Health 57:141-146

5. Barker DJP (1998) Mothers, babies and health in later life. Churchill Livingstone, London

6. Lawlor DA, Ebrahim S, Davey Smith G (2002) A lifecourse approach to coronary heart disease and stroke. In: Kuh D, Hardy R (eds) A life course approach to women's health. Oxford University Press, Oxford, pp 86-120

7. Davey Smith G, Ben-Shlomo Y, Lynch J (2002) Life course approaches to inequalities in coronary heart disease risk. In: Stansfeld S, Marmot M (eds) Stress and the heart: psychosocial pathways to coronary heart disease. BMJ books, London, pp 20-49

8. Strachan DP (1997) Respiratory and allergic diseases. In: Kuh D (ed) A life course approach to chronic disease epidemiology. Oxford University Press, Oxford, pp 101-120

9. Dezateux C, Stocks J (1997) Lung development and early origins of childhood respiratory illness. Br Med Bull 53:40-57

10. Sherrill DL, Lebowitz MD, Knudson RJ, Burrows B (1991) Smoking and symptom effects on the curves of lung function growth and decline. Am Rev Respir Dis 144:17-22

11. Frayling TM, Hattersley AT (2001) The role of genetic susceptibility in the association of low birth weight with type 2 diabetes. Br Med Bull 60:89-101

12. Hales CN, Barker DJ (2001) The thrifty phenotype hypothesis. Br Med Bull 60:5-20

13. Lawlor DA, Ebrahim S, Davey Smith G (2002) The association between components of adult height and Type II diabetes and insulin resistance: British Women's Heart and Health Study. Diabetologia 45:1097-1106

14. Lawlor DA, Ebrahim S, Davey Smith G (2002) Socioeconomic position in childhood and adulthood and insulin re- sistance: cross sectional survey using data from British women's heart and health study. BMJ 325:805-807

15. Engstrom G, Hedblad B, Nilsson P, Wollmer P, Berglund G, Janzon L (2003) Lung function, insulin resistance and incidence of cardiovascular disease: a longitudinal cohort study. J Intern Med 253:574-581

16. Lange P, Groth S, Kastrup J et al. (1989) Diabetes mellitus, plasma glucose and lung function in a cross-sectional population study. Eur Respir J 2:14-19

17. Mori H, Okubo M, Okamura M et al. (1992) Abnormalities of pulmonary function in patients with non-insulin-dependent diabetes mellitus. Intern Med 31:189-193

18. Lazarus R, Sparrow D, Weiss ST (1998) Impaired ventilatory function and elevated insulin levels in nondiabetic males: the Normative Aging Study. Eur Respir J 12:635640

19. Marvisi M, Bartolini L, Borrello P del et al. (2001) Pulmonary function in non-insulin-dependent diabetes mellitus. Respiration 68:268-272

20. Engstrom G, Janzon L (2002) Risk of developing diabetes is inversely related to lung function: a population-based cohort study. Diabet Med 19:167-170

21. Walter RE, Beiser A, Givelber RJ, O'Connor GT, Gottlieb DJ (2003) Association between glycemic state and lung function: the Framingham Heart Study. Am J Respir Crit Care Med 167:911-916

22. Lawlor DA, Bedford C, Taylor M, Ebrahim S (2003) Geographical variation in cardiovascular disease, risk factors, and their control in older women: British Women's Heart and Health Study. J Epidemiol Community Health 57: 134-140

23. Higgins MW, Keller JB (1973) Seven measures of ventilatory lung function. Population values and a comparison of their ability to discriminate between persons with and without chronic respiratory symptoms and disease, Tecumseh, Michigan. Am Rev Respir Dis 108:258-272

24. Trinder P (1969) Determination of blood glucose using 4amino phenazone as oxygen acceptor. J Clin Pathol 22:246

25. Andersen L, Dinesen B, Jorgensen PN, Poulsen F, Roder ME (1993) Enzyme immunoassay for intact human insulin in serum or plasma. Clin Chem 39:578-582

26. Matthews DR, Hosker JP, Rudenski AS, Naylor BA, Treacher DF, Turner RC (1985) Homeostasis model assessment: insulin resistance and beta-cell function from fasting plasma glucose and insulin concentrations in man. Diabetologia 28:412-419

27. Ferrara CM, Goldberg AP (2001) Limited value of the homeostasis model assessment to predict insulin resistance in older men with impaired glucose tolerance. Diabetes Care 24:245-249

28. World Health Organisation (1999) Definition, diagnosis and classification of diabetes mellitus and its complications. Report of a WHO Consultation. Part 1: Diagnosis and classification of diabetes mellitus. WHO, Geneva

29. Office of Population Censuses and Surveys (1980) Classification of occupations and coding index. HM Stationary Office, London

30. Cole TJ (1975) Linear and proportional regression models in the prediction of ventilatory function. J R Stat Soc Ser A 138:297-324

31. Davey Smith G, Greenwood R, Gunnell D, Sweetnam P, Yarnell J, Elwood P (2001) Leg length, insulin resistance, and coronary heart disease risk: the Caerphilly Study. J Epidemiol Community Health 55:867-872

32. Gunnell D (2002) Can adult anthropometry be used as a 'biomarker' for prenatal and childhood exposures? Int J Epidemiol 31:390-394 
33. Erens B, Primatesta P (1999) Health Survey for England 1998: Cardiovascular disease. HM Stationary Office, Department of Health, London

34. Allen DS, Ellison GT, Santos Silva I dos, De Stavola BL, Fentiman IS (2002) Determinants of the availability and accuracy of self-reported birth weight in middle-aged and elderly women. Am J Epidemiol 155:379-384

35. Fall CH, Osmond C, Barker DJ et al. (1995) Fetal and infant growth and cardiovascular risk factors in women. BMJ 310:428-432

36. Burrows B, Bloom JW, Traver GA, Cline MG (1987) The course and prognosis of different forms of chronic air- ways obstruction in a sample from the general population. N Engl J Med 317:1309-1314

37. Thomason MJ, Strachan DP (2000) Which spirometric indices best predict subsequent death from chronic obstructive pulmonary disease? Thorax 55:785-788

38. Wannamethee SG, Shaper AG, Perry IJ (2001) Smoking as a modifiable risk factor for type 2 diabetes in middle-aged men. Diabetes Care 24:1590-1595

39. Lange P, Parner J, Schnohr P, Jensen G (2002) Copenhagen City Heart Study: longitudinal analysis of ventilatory capacity in diabetic and nondiabetic adults. Eur Respir J 20:1406-1412 